\title{
Layer-Two Interference Control Schemes for LTE Systems
}

\author{
Jun Chen ${ }^{1, a}$ \\ ${ }^{1}$ School of Aeronautics and Astronautics, University of Electronic Science and Technology of China, \\ Chengdu 611731, China \\ a2050305649@qq.com
}

Keywords: Interference, Layer-Two, cellular system, Interference Control, simulation and analysis.

\begin{abstract}
In the modern times, there exists large-scale electromagnetic interferences and confrontations. If the communication device and the communication technology do not have a very good anti-jamming capability, the communication quality will not be guaranteed. Firstly, this article descripts the rationale to do interference control in LTE systems. Secondly, identifies solutions of interference control for LTE systems. At last, analysis the performance of candidate algorithms and simulates them, and gets a satisfactory result.
\end{abstract}

\section{Introduction}

This document presents the functional principles of Layer-Two Interference Control function defined for the concepts of Radio Resource Management (RRM) for LTE systems. The definition and requirements of the LTE systems can refer to [1][2] .

\section{Rational to Do Interference Control in LTE Systems}

The current working assumption of maximum data rates for a LTE cellular system results in a high frequency bandwidth requirement. ${ }^{[3]}$ To achieve reasonable multi-operator scenarios with limited total bandwidth availability, the frequency re-use factor in the network must be low. With very low frequency-reuse, other-cell or inter-cell interference becomes serious at the sector boundary or at the cell edges.

There has been an interference study [4] for 4G systems with the assumption of a Time Division Multiple Access (TDMA) system and full-load situation. For the Band Width (BW) of $12.8 \mathrm{MHz}$ with frequency-reuse of 1/3, 50\% coverage at Down Link (DL) has the Signal to Interference plus Noise Ratio (SINR) above 13-14 dB and 90\% coverage at DL has the SINR above 2-3 dB. 12.8 $\mathrm{MHz}$ is similar to the BW requirements of LTE systems. It implies that certain interference control schemes are required for achieving acceptable coverage and a proper region where Multiple-Input Multiple-Output (MIMO) works.

\section{Candidate Algorithms for Interference Control}

\subsection{Overview of the Proposed Interference Control Schemes}

\subsubsection{Definition of Loading}

To facilitate the explanations of proposed IC schemes, the loading is defined as the ratio of used resources (e.g., time/frequency slots occupied by data traffic) to the whole resource pool (e.g., the scheduling period/band).

In general, we assume that cell loading is measured and reported at each cell. To facilitate the explanations, we use time/frequency slots for "resources" in the following sections.

\subsubsection{How the Proposed IC Schemes Work Together}

As LTE system employs a FDMA/TDMA multiple access scheme, the available resources for interference control are thought as: (1) Time. (2) Frequency. (3) Power.

Time and frequency can be thought as "orthogonal" resources. If different time and frequency slots are used at different cells, other-cell interference is limited due to time/frequency isolation. Before adjusting power to control interference, time/frequency isolation should be used. This idea is 
better suited for low-loading situation.

On the other hand, power is not "orthogonal” resources. Adjusting power can control interference, but except using zero power there always be interference. Adjusting power is better suited for high-loading situation. With the aforementioned thinking, the proposed interference control schemes can be categorized into two types: schemes for low-loading situation and schemes for high-loading situation.

\subsection{Interference control Schemes for Low-loading Situation}

\subsubsection{Adaptive Reuse-Pattern-based Scheduling Algorithm}

Packet scheduler in each cell follows a guide of the preconfigured reuse patterns so that the inter-cell interference can be suppressed. ${ }^{[6]}$ A set of several reuse patterns are prepared for different load conditions, and the reuse pattern is adaptively chosen from this set based on the operational load condition. The packet scheduler first schedules the capacity requests to the time/frequency slots that the chosen reuse pattern indicates. If the packet traffic cannot be fully accommodated in the slots that the reuse pattern indicates, the packet scheduler schedules the excess amount of traffic in other slots. (only this excess amount of traffic generates the interference).

\subsubsection{Coordinated Scheduling for Load Balancing}

This algorithm proposes a dynamic coordinated scheduling (or channel allocation) method for the following purposes:

(1)For a set of cells, obtain the trunking gain by combining the time/frequency slots at each cell as a big resource pipe/pool for scheduling.

(2)For a set of cells, obtain the traffic diversity by exploring different traffic characteristics at different cells.

The algorithm can be implemented in either the distributed way or the centralized way. In the distributed way, the coordinated scheduling is done at the site where several Base Transceiver Station (BTSs) are co-sited. In other words, the set of cells is the set of co-sited BTS. In this case, there is no need for each cell to report its loading information.

In the centralized way, this function is located at one central network element .The set of cells is controlled by RRO. Every cell in the set reports its loading information to the RRO. After the coordinated scheduling, RRO will then inform the detailed decisions to each cell.

\subsection{Interference Control Schemes for High-loading Situation}

\subsubsection{Power Sequence Based Interference Control Scheme}

To tackle the inference problems in a low frequency-reuse scenario, a novel solution ${ }^{[5]}$ has been proposed in 2004.

To apply this method in the LTE systems, there are several points we should highlight:

(1) The simplest way is to apply power-sequence in the frequency domain so that the mutually synchronized BSs are not requited. If applying power-sequence in time-domain, the effects of synchronization errors on the performance will be studied via simulations.

(2) If applying power sequence in frequency domain, it is difficult to predict C/I or SINR at subcarriers or sub-bands (grouped subcarriers). In this case, we know the power sequence at frequency domain, $\mathrm{P}(\mathrm{f}) . \mathrm{C}(\mathrm{f})=\mathrm{P}(\mathrm{f}) / \mathrm{L}(\mathrm{f})$, where $\mathrm{L}(\mathrm{f})$ is a product of loss, shadowing and fast-fading. This leads to the $\mathrm{C} / \mathrm{I}$ function at frequency-domain as: $\mathrm{C} / \mathrm{I}(\mathrm{f})=\mathrm{C} 1(\mathrm{f}) / \mathrm{sum}(\mathrm{Cn}(\mathrm{f}))$, cell 1 is serving cell. If we intend to predict $\mathrm{C} / \mathrm{I}(\mathrm{f})$, we have to know $\mathrm{L}(\mathrm{f})$ from the surrounding BSs.

(3) We can still keep the power sequence, but we may not be able to predict C/I(f) for next scheduling period. Please note that the algorithm still works even without the predictive feature.

\section{Perfomance Studies of Interference Control Algorithms}

\subsection{Simulations}

In the simulator, free space propagation model and Okumura-Hata propagation model are implemented ${ }^{[7]}$. The following figure shows the pathloss generated from these two models. In all the simulations mentioned in this document is performed by using Okumura-Hata model. ${ }^{[8][9]}$

Because the traffic generation is a stochastic process, the variation of the amount of the traffic has an impact on the performance of scheduling algorithm. Only the way to control this variation for the 
system is the length of the scheduling period. If the scheduling period is large, the ratio of traffic amount variation to the traffic amount becomes small. Then, the performance of the scheduling algorithm would be better. The following figure shows an example of this case. The simulation is performed by using reuse-9-type scheduling algorithm. From this result, other simulations on this document are performed with 18 slots.

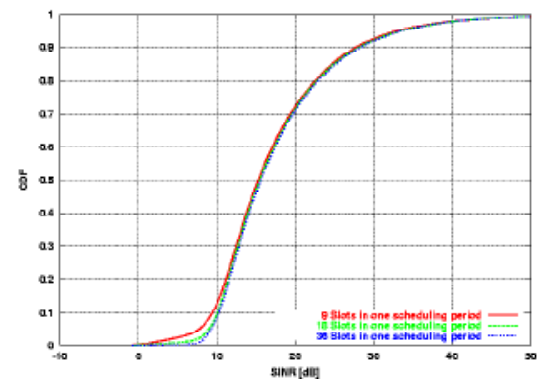

Fig. 1: Effect of the number of resource slots in one scheduling period

For the comparison of interference control algorithms, the following 5 cases are simulated. ${ }^{[10]}$

(1) Conventional frequency reuse $1 / 3$ system

Frequency reuse $1 / 3$ is used, and only one-third of the available spectrum can be utilized at maximum.

(2) Random scheduling algorithm for frequency reuse 1 system

No intelligent scheduling algorithm is applied. Traffic is just randomly allocated to available resource. In other word, no interference control is intentionally applied.

(3) 1/3-reuse-pattern PS for frequency reuse 1 system

Similar to the conventional reuse 1/3 system, cells are classified into three groups. Based on this classification, it is pre-determined that by what order, the available resources of the first one-third, the middle one-third, and the last one-third are used for scheduling. Compared with conventional $1 / 3$ reuse method, all resource slots can be used for scheduling and excess traffic will be scheduled in "non-orthogonal slot", as explained in section 6.2.1, although this traffic may generate interference.

(4) $1 / 9$ to $1 / 3$ adaptive-reuse-pattern PS for frequency reuse 1 system

This is an extension of 1/3-reuse-pattern PS in two senses. One is that it is extended to 1/9-reuse-pattern, which would be beneficial for low load situation. The other is the adaptiveness to the system load. If the load is high, PS switches to 1/3 reuse-pattern PS. Note that the system load is not an input parameter but measured at each cell. This algorithm adaptively changes the scheduling scheme based on the system load (amount of capacity request).

(5) Power-Sequence-based Dynamic Channel Assignment (DCA)

In general, there can be as many power levels as the number of resource slots in one power sequence. For example, if there are 18 resource slots in one scheduling period, one power sequence can have 18 power levels in it. In the simulator, however, a simple power sequence scheme has been implemented. In this scheme, only three power levels are available in one power sequence. Namely, in the above example, every 6 resource-slots use the same power level.

To evaluate the performance effects of IC algorithms for different system load situation, simulations are performed for 33\% and $80 \%$ load cases.

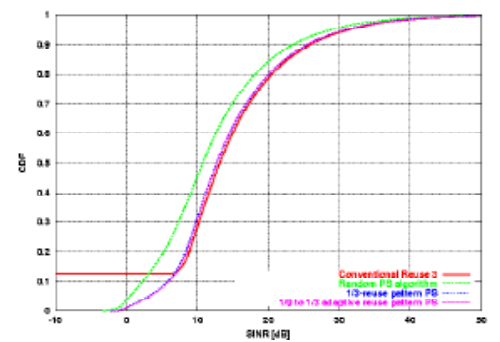

Fig. 2: Comparison of different algorithms at $33 \%$ load 


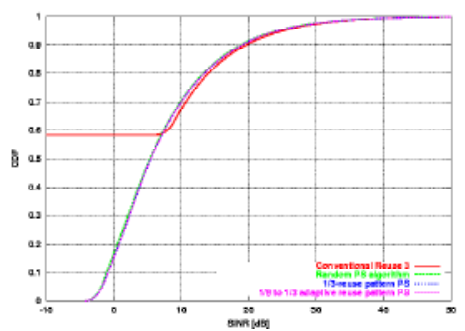

Fig. 3: Comparison of different algorithms at $80 \%$ load

\subsection{Evaluation of Power-sequence-based Dynamic Channel Assignment} 4.2.1 Performance at Different System loads

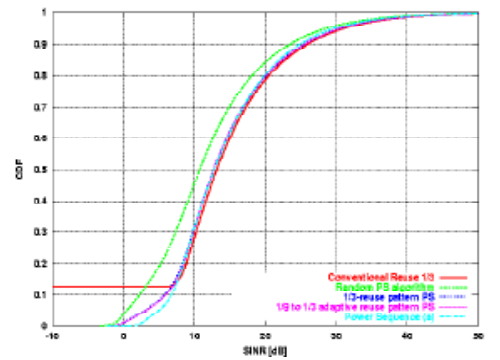

Fig. 4: Comparison of different algorithms at $33 \%$ load

At 33\% load, power-sequence-based DCA achieves almost the same SINR performance in high SINR region ${ }^{[11]}$, and the highest SINR in low SINR region. This is a gain available by using power-sequence-based DCA.

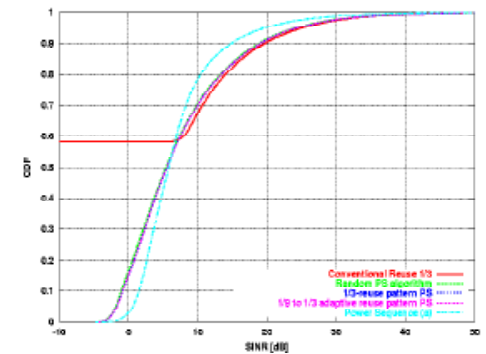

Fig.5: Comparison of different algorithms at $80 \%$ load

At $80 \%$ load, different from other flat-power scheduling algorithms, power-sequence-based PS considerably improves the SINR performance. For example, if $0 \mathrm{~dB}$ is required for maintaining cell coverage, flat-power scheduling algorithms cannot serve for about 15\% users, whereas power-sequence-based DCA can decrease it to less than 5\%.

\section{Conclusions}

Observing from the simulation results, it can be concluded that:

(1) In low load situation, frequency/time-based DCA, "adaptive-reuse-pattern Packet Scheduling algorithm”, can achieve the considerable improvement in both low and high SINR region. Therefore this can be utilized for improving QoS/throughput performance in addition to improving cell coverage.

(2) With the change of the load, the reuse pattern should be adaptively changed.

(3) In high load situation, other mechanism such as power based DCA is required. According to the simulation results, there is considerable gain available, especially the improvement at low SINR region.

(4) By designing power sequence properly, interference can be controlled.

\section{References}

[1] Jussi Kahtava, “3.9G Physical Layer Specification”, in 3.9G Physical Layer Spec V0.1.9.doc, March 28, 2005.

[2] 3GPP TR25.913 v0.0.1, “Requirements for Evolved UTRA and UTRAN”, May, 2005. 
[3] H. Zhu, K. Leppanen, S. Savio, "Power-sequence-based predictive and adaptive interference control and packet scheduling scheme”, NC37574, accepted on Oct. 21, 2004.

[4] Jussi Ojala, "Initial Coverage and Interference Analysis of Outdoor 4G-network Using a Static Simulator”, deliverable of MRR project, version 3.0, Dec. 16, 2003.

[5] The Third Generation Partnership Project (3GPP) E-UTRA and E-UTRAN Overall description; Stage 2(Release 8),3GPP Technical Specifications TS 36.300 V8.12.0 2011.

[6] SongBo He, "Broadband wireless communication transceiver and key technologies”[D]. CHENDU : University of Electronic Science and Technology of China.(in Chinese)

[7] H. Zhu, K. Leppanen, S. Savio, "Power-sequence-based predictive and adaptive interference control and packet scheduling scheme”, NC37574, accepted on Oct. 21, 2004.

[8] TSG RAN WG1, Tdoc \#R1-050443, “Simulation Assumptions and Evaluation for EUTRA”, May, 2005

[9] YuBin Shao.” The study on Communications system modeling and simulation. ” [M]. BEIJING: Tsinghua University Press, 2007:62-85.(in Chinese)

[10] Stabellini. L, Shi. L, Al Rifai. A, Espino. J, Magoula. V, "A new probabilistic approach for adaptive frequency hopping", Personal, Indoor and Mobile Radio Communications, 2009 IEEE 20th International Sympousium on, on page(s):2147-2151, Sept 2009.

[11] Ksairi N;Bianchi P;Ciblat P Resource allocation for downlink cellular OFDMA systems-Part I :Optimal allocation , 2010(02).

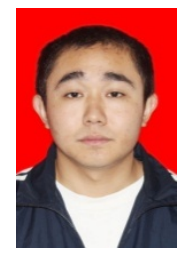

Chen Jun was born in Sichuan Province, China, in 1989. Now he is studying in University of Electronic Science and Technology of China, Chengdu. He is a second-year graduate student in School of Aeronautics and Astronautics. His major is Navigation Guidance and Control and His research interests include wireless communication and mobile communication and control systems.

Tel : 15388363885 